
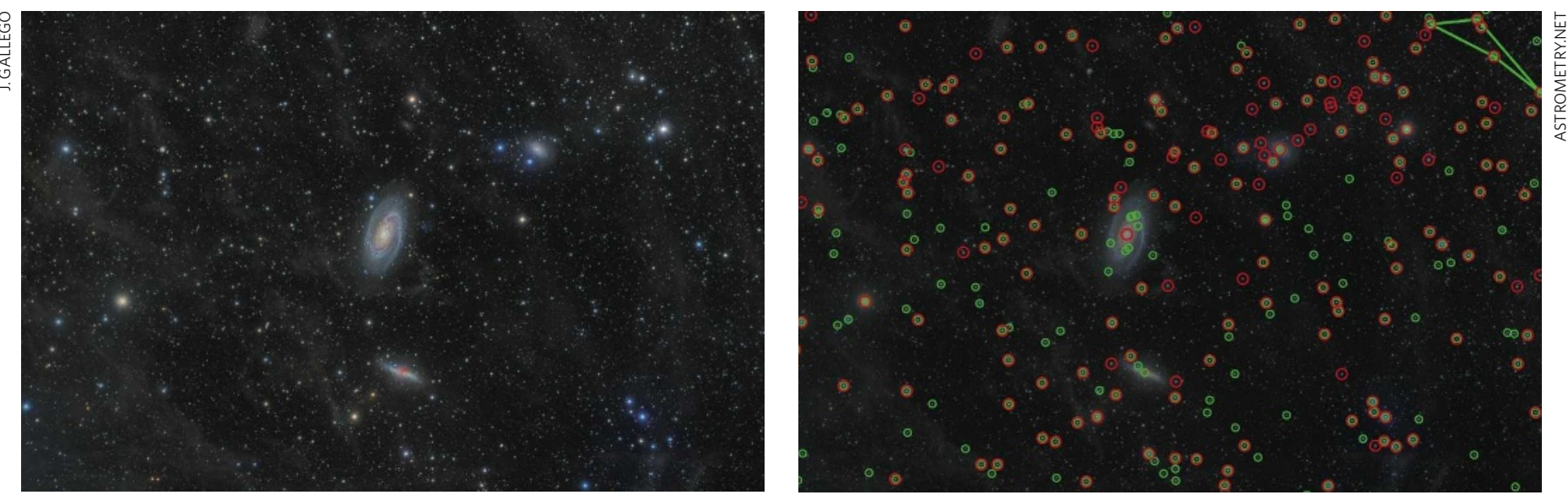

Left, the sky around galaxy M81; right, Astrometry.net uses a recognized shape (top right) to line up stars in the image (red) with those in its memory (green).

\title{
No star left behind
}

\section{An open-source software project could help unify every existing astronomical image into a single data set.}

Everyone knows the experience: a pile of old photos in a dusty attic - or more likely now on a dusty disk drive - with no indication of who is in them or when they were taken. As long as the subjects are astronomical features, though, there's now an answer at hand.

Astrometry.net is an open-source software project, run out of the University of Toronto in Canada and New York University, which aims to recognize any starscape and place it in its proper coordinates within seconds - specifying not just which patch of the sky is shown, but when. By using the small relative motions of stars over time, the project's designers hope to date any picture to within a year.

The project should regularize astronomical data, making it possible to combine images from both professionals and amateurs into easily used databases. "We'd love to touch every astronomical image ever taken," says David Hogg, a project leader and an astrophysicist at New York University. The system is currently undergoing initial alpha-testing by astronomers at various observatories, but there are plans to start accepting images from the public by September.

Although astronomers try to use a universal coordinate system to chart the sky, the reality is a messier business: telescope pointing mechanisms are idiosyncratic and not always properly used. This can make data from different observatories difficult to compare. Astrometry.net solves the matching problem by choosing bright, four-star constellations from the pixels in a submitted image. It then looks for the same shapes in an index of 800,000 such constellations constructed from all-sky surveys catalogued by the US Naval Observatory.

In a test, the software gave the correct coordinates for all but 451 of 336,554 images from the Sloan Digital Sky Survey, a systematic map of a quarter of the sky. The team has focused on speed and reliability: all of the matches were made in less than a second, and there were no false positive matches.

Tim Axelrod, the data management project scientist for the Large Synoptic Survey Telescope, says it's the most robust calibration system he has seen, and he plans to use it when his telescope begins its surveys in 2014 (the system is powerful enough to survey the entire night sky in three days).

As the Naval Observatory catalogues were made from multiple sky surveys done about 30 years apart, they document tiny shifts in the positions of nearby stars, called their proper motions. This means that, once Astrometry.net has its match, it can turn back the clock, rewinding the proper motions to the best match possible for the pattern documented in a submitted image. Astronomers will thus be able to pick out features that have changed over time, such as Kuiper-belt objects in the Solar System and supernova precursors.

Jonathan Grindlay, a researcher at Harvard University who is interested in transient phenomena, is eager to use the system. He runs a programme aimed at digitally scanning more than half a million photographic plates dating back to the 1880 s that, between them, cover every inch of the sky between 500 and 2,000 times. Astrometry.net should go a long way to sorting out exactly what each plate records, and when it was taken. Once it does, a century of the skies will be open to scrutiny for changes on timescales of decades. Eventually, Hogg would like the software to recognize any unusual feature as part of the system's routine service. But there are pressing wrinkles to iron out first. An image needs enough four-star constellations for the software to make a match, and so the system has trouble with pictures that cover less than one ten-millionth of the sky. This is an issue for images from instruments such as the Hubble Space Telescope, which looks very deeply into tiny patches of the sky. And to get the most out of the system, the software will need to match images, not just in space and time, but also in brightness - the apparent brightening and dimming of a star, for instance, would have to be referenced to something.

But as the software improves, it could become the standard for the entire community, which is trying to supply streams of data to portals such as the planned international 'virtual observatory'. Already, the US National Optical Astronomy Observatory in Tucson, Arizona, plans to be using Astrometry.net by the end of the year to calibrate the 12 terabytes of data it collects annually from its 12 telescopes.

If it ends the laborious days of calibrating astronomical data by hand, Astrometry.net would be saving not only lost photons, but also precious time and money, says Hogg. "The amount of money this would save the astronomical community is immense," he says. Eric Hand

See Editorial, page 428.

For further information, see http://tinyurl. com/6y3bkv 


\section{Beep-beep! Roadrunner breaks petaflop barrier}

The US military's Roadrunner supercomputer has become the first machine to calculate at more than a quadrillion $\left(10^{15}\right)$ operations per second, thereby crossing the petaflop barrier.

The record was technically set on 25 May, in IBM's laboratories in Poughkeepsie, New York, using the LINPACK standard benchmark for comparing supercomputer performance. In early June, however, it achieved petaflop performance on a real-life application of modelling the human cortex, says David Turek, vice-president for 'deep computing' at IBM.

It thus beats the record of IBM's Blue Gene/L machine, based at the Lawrence Livermore National Laboratory in California, which managed about 500 teraflops $\left(10^{12}\right)$.

Roadrunner was built to run calculations to ensure the safety of the US nuclear stockpile, and is due to be delivered next month to the Los Alamos National Laboratory in New Mexico for that purpose. In the meantime, it will also run other calculations, including plasma physics, molecular dynamics and climate change.

\section{Physicist's involvement in historical race row disputed}

A historical investigation has posthumously cleared Eligio Perucca, a physics professor at the Polytechnic University of Turin, of alleged anti-semitism towards Primo Levi.

Levi, an Italian chemist, writer and

Auschwitz survivor, described in his book The Periodic Table the difficulties he had faced in persuading several professors in Turin to take him on as a doctoral candidate in 1940, because of the race laws that the Fascist regime

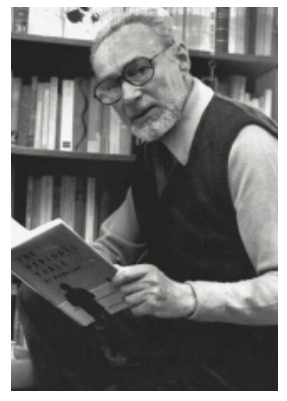

Primo Levi. imposed in Italy at that time. Perucca was later fingered as one of these professors in a Levi biography.

But Bart Kahr, a chemist at the University of Washington in Seattle, has looked into the claim and deduced that Perucca was not a fascist nor an anti-semite.

What's more, the investigation credits Perucca with a chemical discovery - of a certain type of optical activity, called optical rotary dispersion, of dyed chiral sodium chlorate crystals - in 1919 that was thought to have been discovered only in 1931.

\section{Japanese lab installed on space station}

Astronauts installed the main piece of Japan's laboratory module, Kibo, aboard the International Space Station last week.

At 11 metres long, Kibo, delivered by the space shuttle Discovery and opened by Japanese astronaut Akihiko Hoshide (pictured), is the largest pressurized research facility in space. Its final section, for work in unpressurized environments, is slated to be flown up next year. Astronauts also tested Kibo's robotic arm, which will be used to manipulate experiments on that final section when installed.

The station's malfunctioning toilet was also fixed during the trip.

\section{Panel urges further review of controversial NIH lab}

A blue-ribbon panel last week told the National Institutes of Health (NIH) that it needs to conduct more studies if it hopes to see high-risk biosafety research conducted at a lab under construction at Boston University Medical Center in Massachusetts.

The panel, chaired by molecular biologist Adel Mahmoud of Princeton University in New Jersey, said that the agency should conduct risk assessments for 12 dangerous pathogens, including comparing the risks of each being released at urban, suburban and rural sites.

Advisers to NIH director Elias Zerhouni, who received the report on 6 June, offered some sharp criticism of the \$198-million project, most of which is being paid for by the NIH. Community concerns about the risks of the facility have led to it being challenged in both the state and federal courts.

\section{Publications follow policy on stem-cell research}

Countries with less restrictive policies for deriving human embryonic stem cells produce a disproportionate share of the field's publications, according to a new study (A. Levine Cell Stem Cell 2, 521-524; 2008).

By this measure, the United States was the worst performer: although $36 \%$ of scientific publications on human embryonic stem cells in 2006 had a US-based corresponding author, that compared with $46 \%$ of a control set of biomedical publications and $47 \%$ of publications on RNA interference, a less controversial 'hot' technology.

The study's author, bioethicist Aaron Levine of the Georgia Institute of Technology in Atlanta, notes that governments in the top five overperforming countries
(Singapore, United Kingdom, Israel, China and Australia) support research and permit derivation of new cell lines. Besides the United States, other underperformers included Japan, France and Switzerland. Both France and Japan also have less than the expected share of publications on RNA interference, and Levine suggests that scientists in those countries are less inclined to pursue emerging technologies.

\section{Senate climate debate suffers death by hot air}

Democratic leaders in the US Senate walked away empty-handed last week after a highly anticipated debate over climate-change legislation disintegrated into partisan bickering and delaying tactics.

The bill, which would have required a two-thirds cut in greenhouse-gas emissions by 2050 , died on 6 June on a procedural vote. Not a single amendment was considered, and no substantive votes were cast.

Opponents said the legislation would further increase energy prices and push US jobs overseas. The lowlight came as Republicans invoked an obscure Senate rule to force staff to read the entire 492-page bill aloud over the course of some nine hours.

Few had expected the legislation to pass, but Democrats and environmentalists had hoped for a substantive debate that would lay the groundwork for next year.

\section{Corrections}

The News story 'No star left behind' (Nature 453,$437 ; 2008$ ) misstated the number of constellations constructed for an index in starmatching software. The number of constellations is 800 million not 800,000 .

In the News story 'Climate anomaly is an artefact' (Nature 453, 569; 2008) we incorrectly stated that David Thompson was at the State University of Colorado in Boulder. He is in fact at the Colorado State University in Fort Collins. 